\title{
Análisis psicológico de la incontinencia urinaria femenina
}

\author{
Antonio Lomanto Morán*, Jacinto Sánchez Angarita*; Beatriz Y. Rochel Navarro**
}

\begin{abstract}
RESUMEN: El objetivo de la investigación es la aplicación de un programa de Intervención Psicológica que identifique e intervenga factores que dificulten la adherencia al tratamiento médico prescrito a pacientes con incontinencia urinaria en el Instituto Materno Infantil (I.M.I.). Los sujetos fueron 80 mujeres de estrato socioeconómico medio-bajo, con edades entre los 30 y 70 años que presentaban el cuadro. Se hizo uso de un diseño cuasi experimental de dos grupos, experimental y control. La variable independiente fue el paquete terapéutico que fomentó la adherencia al tratamiento. El análisis de los resultados permite establecer características de acuerdo con cada uno de los grupos diagnósticos en cuanto a los niveles de neuroticismo, ansiedad, depresión y estilos de afrontamiento. Igualmente se estableció la eficacia del programa psicológico para la identificación y manejo de las diversas variables que afectan el cumplimiento.
\end{abstract}

PALABRAS CLAVES: Incontinencia urinaria, neuroticismo, ansiedad y depresión.

SUMMARY: The objetive, was to applicate a program of psichologic counseling to identify and change factors affecting the acomplishment of medical treatment of stress incontinence patients at the Instituto Materno Infantil (I.M.I.) 80 midle-low income patients between 30 and 70 years old were studied. The study was cuasiexperimental in two groups: Experimental and controls. The independent variable was the therapeutic package used to imprave adherence to treatment. The results analysis, establishes features according to each one. Of the diagnostic groups regarding neurotic, onxiety, depression and facing modalities levels. The efficiency of the psychologic program in the identification and management of the several variables affecting acomplisment, was also measured.

KEY WORDS: Stress incontinence, neuroticism, anxiety and depression.

\section{Introducción}

La Clínica de Incontinencia Urinaria del I.M.I., atiende población femenina que reporta evidencia de dicha problemática y que en su mayoría son remitidas por otros servicios de la misma institución para la valoración e intervención médica.

Se ha observado que la dificultad en la retención de la orina en muchos casos está asociada a estados depresivos y/o ansiógenos en la mujer, independientemente de patología orgánica, o aún cuando esta involucrada, puede verse disminuida si se combina con una intervención médica o psicológica (Mackaulay, Stern, Holmes, Stanton, 1987).

El diagnóstico en trastornos de la micción se hace teniendo como base la historia clínica, el examen físico y análisis de laboratorio, a partir de los cuales se identifican aspectos patológicos al tracto urinario, desórdenes neurológicos, desórdenes psiquiátricos efectos locales de enfermedades sistémicas y su tratamiento, frecuencia de micción, antecedentes gineco-obstétricos, estado físico general (diabetes), pruebas de incontinencia, peso, estatura, lo que permite establecer el tipo de incontinencia que padece la paciente (incontinencia urinaria de esfuerzo IUE, Incontinencia Urinaria de Urgencia, IUU, Incontinencia urinaria Mixta, IUM) y el tratamiento más adecuado según las características específicas del caso.

* Profesores Asociados. Departamento de Ginecología y Obstetricia. Departamento de Psicología. Universidad Nacional de Colombia.
Dado que este trabajo enmarca dentro de los lineamientos teóricos de la Psicología de la salud cuyo objetivo es promover la salud y prevenir la enfermedad, la intervención psicológica tiene como tarea fundamental la identificación de factores que puedan estar predisponiendo a la patología o aquellos que la estén manteniendo, es decir, a través del trabajo psicológico se establecen estilos de vida, elementos comportamentales, costumbres, creencias de inutilidad del tratamiento, aspectos emocionales que posibiliten o desencadenen el problema y la adherencia al tratamiento por incomprensión de instrucciones médicas, creencias, depresión, ansiedad, baja auto-estima, respectivamente. De esa forma, se ayuda a la paciente a desarrollar estímulos de afrontamiento acordes a su dificultad, comportamientos autocontrolados, solución de problemas que le permitan un desempeño más adecuado con el fín de promover y mantener su salud integral, dado que la incontinencia urinaria puede afectar su vida de pareja, su vida laboral, económica y social.

Muchas encuestas arrojan datos que indican que aproximadamente $50 \%$ de mujeres sanas jóvenes presentan alguna pérdida involuntaria de orina, pero sólo se reconoce como patología y se acude al médico cuando la pérdida ocurre con mayor frecuencia y es casi imposible de controlar en cualquier situación (Lomanto, 1989).

La Asociación Nacional sobre Continencia define la incontinencia Urinaria (IU) como "una condición en la que la salida de la orina constituye un problema social o higiénico y es objetivamente demostrable" (Abrams, Blavias, Stanton, 1988) Así mismo, se le clasifica en 
pérdida involuntaria de la orina. Incontinencia urinaria Intermitente (eneuresis, incontinencia de urgencia, incontinencia esfuerzo, incontinencia de sobredistensión, incontinencia funcional) e incontinencia urinaria continua (incontinencia congénita, traumática o neurógena).

Se consideran factores etiológicos el trauma obstétrico, las alteraciones de tipo hormonal, la atonía miouretral, la latrogénica operatoria y factores raciales. (Lomanto, 1989).

Dado que la IU es una patología que puede estar asociada a diferentes factores, es necesario observar durante la evaluación clínica todos aquellos aspectos que ayuden a precisar el diagnóstico: Historia familiar de diabetes sacarina, historia neurológica, historia ginecoobstétrica, historia psiquiátrica, historia farmacológica, antecedentes quirúrgicos, antecedentes urológicos, examen físico, sensibilidad del periné, examen ginecourológico, pruebas de incontinencia, pruebas de esfuerzo, procedimientos paraclínicos.

De esta forma se puede establecer con mayor precisión el tipo de intervención que debe fijarse para el manejo de la IU. El tipo de procedimiento elegido depende de las particularidades del caso. Se hace uso de intervenciones quirúrgicas cuando el médico por valoración clínica comprueba su carácter necesario.

En caso contrario, se pueden establecer tratamientos farmacológicos, de estimulación eléctrica funcional, acupuntura, hipnosis, adiestramiento en rehabilitación vesical, biorretro alimentación o ejercicios de contracción de los músculos perineales. En el Instituto Materno Infantil, se recurre a este último método, dado que se puede emplear en cualquier consultorio ginecológico ya que no requiere de implementos de laboratorio especializados. Arnold Kegel en la década de los 40 fue el primero en señalar la eficacia de los ejercicios de los músculos del suelo perineal para el tratamiento de la IUE, haciendo innecesaria la intervención quirúrgica. El fundamento de este tratamiento es la observación de la recuperación del tono de los músculos debido a la contracción voluntaria, llevando finalmente a la recuperación de la capacidad esfinteriana uretral.

Como puede observarse, el tratamiento establecido requiere las estrategias de autocontrol que permita a las pacientes adoptar una posición activa frente a su condición de salud, es decir, que la recuperación depende en gran medida de su colaboración. Para tal fin se entrena a la persona a incorporar dentro de su repertorio comportamental principios de la teoría del moldeamiento para que se aumente el número de contracciones diarias de forma progresiva, entrenamiento en control de estímulos que permitan recordar la ejecución del tratamiento y estrategias del autoregistro y contratos sociales.

El objetivo final del tratamiento es establecer que la paciente con incontinencia urinaria realice entre 250 y 300 contracciones diarias por lo menos durante tres meses, tiempo en el cual el equipo médico decide si se hace necesaria o no la intervención quirúrgica.

Otro de los aspectos considerables en el tratamiento de la IU es el estado emocional y afectivo de estas pacientes.
La ansiedad y la depresión son dos factores característicos. Makaulay, Stern, Holmes y Stanton (1987) establecieron relaciones entre desórdenes en la micción y factores mentales. Tanto las mujeres con IUE como IUU presentaron altos niveles de ansiedad y depresión haciéndose más evidente en estas últimas. De la misma forma, Herzog, Dioknop y Fultz (1989) al estudiar una comunidad de adultos con IU severa, encontraron altos niveles de ansiedad y depresión, a diferencia de aquellos que no padecían la disfunción. Estas investigaciones coinciden con los reportes realizados por los diferentes profesionales que trabajan en la Clínica de la incontinencia Urinaria del IMI.

La baja auto estima, sentimiento de impotencia, inseguridad, frustración, llevan a la paciente a desarrollar comportamientos poco adecuados frente a su problemática.

Puesto que un mismo problema no afecta en forma equivalente a todos los individuos, debido a que las apreciaciones o valoraciones que las personas hacen de un mismo problema no son las mismas, se hace necesario identificar actitudes, respuestas y soluciones presentadas frente a su problema de salud o frente a cualquier dificultad en general.

La labor que se viene desarrollando en el IMI, pretende establecer factores psico-afectivos que puedan estar involucrados tanto en la etiología como en la evolución de las diferentes enfermedades en el área gineco-obstétrica. (Florez, 1989). Especialmente en el Servicio de incontinencia, las dificultades comúnmente asociadas a este tipo de incapacidad son en resumen, la ansiedad, depresión y poca adherencia al tratamiento requerido para el manejo del problema.

La hipótesis general estima que se fomentará y mantendrá la adherencia al tratamiento prescrito mediante el sostenimiento de las pacientes a un paquete terapéutico que involucra reestructuración cognitiva, suministro de información acerca del manejo y evolución de la patología, y entrenamiento en solución de problemas.

Se hizo uso del siguiente instrumento para establecer una línea base y el impacto de la intervención propuesta:

1. Entrevista estructurada.

2. Hoja de registro del número de ejercicios diarios.

3. Pruebas de ansiedad estado-rasgo, depresión, personalidad y estilos de afrontamiento.

Se sometieron a control las variables edad y tipo de incontinencia para discriminar el efecto de la edad sobre la dificultad para realizar los ejercicios y las características psicológicas específicas de acuerdo al diagnóstico respectivamente.

\section{Método}

\section{Diseño:}

Se aplicó un diseño cuasi-experimental de dos grupos, experimental y de control, con medición pre y post.

\section{Sujetos}

Se tomó una muestra de 80 mujeres que fueron remitidas a la Clínica de Incontinencia Urinaria del IMI a las cuales se les diagnóstico el cuadro y cuyas edades oscilaron entre los 30 y 70 años. 


\section{Técnicas e Instrumentos}

Se procedió a evaluar de la siguiente forma:

1. Aplicación del formato de entrevista inicial estructurada, así como también observación de la hoja de registros de ejercicios realizados diariamente desde la consulta médica a la primera consulta psicológica una semana después.

2. Aplicación del cuestionario de personalidad de Eysenck (1984) y suministro de información sobre el objetivo de los ejercicios y la forma de realizarlos.

3. Aplicación de los inventarios de ansiedad estadorasgo, STAI (Spielberger, 1970) depresión (Beck, 1976) y estilos de afrontamiento (Lazarus y Folkman, 1986 y Vitalino, Maiuro, Pussio y Becker, 1987).

Respecto a la intervención, se establecieron diferentes modelos de acuerdo a lo encontrado en cada caso:

1. Tríada de autocontrol (Cautella, 1984): Para el manejo de pensamientos irracionales o imaginaciones inapropiadas generadoras de ansiedad y/o depresión. Para el caso se tuvo como referencia los puntajes obtenidos en el STAI en la escala ansiedad estado y los puntajes de la escala de depresión leve/moderad de Beck.

2. Técnica ABC (Ellis y Grieger 1981): para el manejo de estados depresivos y altos niveles de ansiedadrasgo.

3. Entrenamiento en relajación autógena, formato reducido propuesto por Shultz (Davis, Mackay y Eshelman, 1985, Escobar y Alarcón, (1990).

4. Entrenamiento en solución de problemas (Mackey, Davisy Fanning, 1987): para el manejo de estados ansiógenos o sentimientos de impotencia generados por ausencia de estrategias para toma de decisiones adecuadas ante una problemática específica.

\section{Procedimientos}

El estudio comprende tres etapas:

1. Línea base, tuvo una duración de tres semanas durante las cuales se identificaron aspectos que pudieran estar dificultando la colaboración con el tratamiento, es decir, altos niveles de ansiedad, depresión, neuroticismo, estilos de afrontamiento inadecuados, desconocimientos del impacto del tratamiento en el organismo.

2. Intervención, etapa en la cual se implementa el tratamiento de acuerdo con las necesidades particulares encontradas.

3. Seguimiento realizado en las dos semanas posteriores al tratamiento psicológico. Esta etapa incluye una entrevista final con el objetivo de determinar el número de ejercicios realizados en el momento y evaluar de nuevo ansiedad y depresión.

\section{Resultados}

El análisis de los resultados se desarrolla en función de los procedimientos de evaluación y las diferencias encontradas entre los niveles de adherencia en los grupos control y experimental.

Adherencia en función de la ausencia o presencia de estrategias comportamentales:
Figura 1

ADHERENCIA EN FUNCION DE LA DEPRESION

Niveles de adherencia según depresión para los grupos control y experimental en la medición pre.

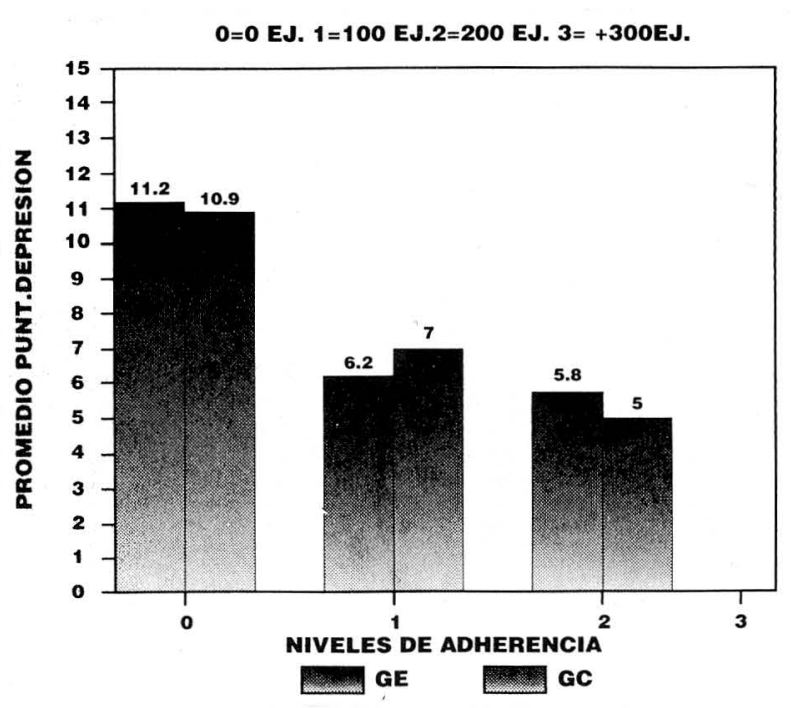

Para la primera semana (Línea base), el $70 \%$ de las pacientes del grupo experimental (GE), reportaron la ausencia de estrategias comportamentales, de igual forma el $95 \%$ de las mujeres del grupo control (GC). Para la última semana (seguimiento), se observó un incremento significativo del número de pacientes de GE con el mayor nivel de adherencia (300 ejercicios diarios) $(\mathrm{Chi}=18.95$, $\mathrm{P}=0.01)$.

En el grupo control (GC) sólo el 5\% de las pacientes se ubicó en el mayor nivel.

En la primera semana, las pacientes más deprimidas del GE alcanzaron los niveles más bajos de adherencia (menos de 100 ejercicios diarios). $(\mathrm{F}=3.52, \mathrm{P}=0.04) 1$ para el grupo control se presenta la misma tendencia pero las diferencias no fueron significativas, $\mathrm{F}=2.4 ; \mathrm{P}=0.1$.

En la última semana se observan diferencias altamente significativas para el GE, a menor depresión, mayor adherencia $(\mathrm{F}=6.33 ; \mathrm{P}=0.01)$. El GC muestra la misma tendencia pero, las diferencias no son significativas, $(\mathrm{F}=1.83 ; \mathrm{P}=$ 0.17). E1 $85 \%$ de las pacientes del GE evidenciaron el mayor nivel de adherencia (300 y más ejercicios diarios). En el GC sólo el 10\% alcanzó este nivel.

\section{Adherencia en función de la ansiedad:}

Para este análisis se toma la ansiedad-rasgo para presentar reducciones significativas en la medición post de GE.

En la primera semana las sujetos menos cumplidoras del GE presentan niveles de ansiedad significativamente mayores que las más cumplidoras $(\mathrm{F}=6.27 ; \mathrm{P}=0.01)$, aunque en el GC las diferencias no son significativas $(\mathrm{F}=$ 2.53; $\mathrm{P}=0.09$ ), se observa la misma tendencia.

En la última semana la mayor parte de las mujeres del GE (34), alcanzaron los niveles más altos de cumpli- 
miento independientemente de los niveles de ansiedad, lo que refleja el efecto homogenizador de la terapia en la reducción del impacto de la ansiedad-rasgo. El GC en cambio, presenta diferencias significativas entre los niveles de ansiedad y el cumplimiento $(\mathrm{F}=3.56, \mathrm{P}=0.04)$, a menor ansiedad, mayor cumplimiento. Se evidencia entonces la necesidad de la terapia para el fortalecimiento de la adherencia.

\section{Adherencia en función de los estilos de afrontamiento:}

Las 34 pacientes del GE son mejores niveles de adherencia (300 y más ejercicios diarios), obtuvieron promedios más altos (1.70) en la escala de estrategias enfocadas en la solución de problemas, que las 6 sujetos menos cumplidoras. A mayor puntaje en solución de problemas, mayor adherencia.

Se observaron claras diferencias entre las estrategias enfocada en la fantasía y la evitación y los niveles de cumplimiento. A mayor puntaje en fantasía y evitación, menor adherencia.

Entre las mujeres del GE la adherencia fue mayor, evidenciando el impacto de la intervención terapéutica independientemente del tipo de afrontamiento.

\section{Adherencia en función de edad:}

Los resultados señalan que no existen diferencias significativas entre los niveles de cumplimiento y la edad de las pacientes en la primera y la última semana. La media de la edad es similar entre las menos y las más cumplidoras, sin embargo tienden a ser más cumplidoras las de menor edad.

Para la primera semana los resultados señalan mayores niveles de neuroticismo en las pacientes poco cumplidoras en ambos grupos. Las diferencias fueron significativas $(\mathrm{GE} . \mathrm{F}=6 ; \mathrm{F}=3.46)$.

\section{Figura 2}

ADHERENCIA EN FUNCION DE LA ANSIEDAD Niveles de adherencia según ansiedad-rasgos para los grupos de control y experimental en la medición pre.

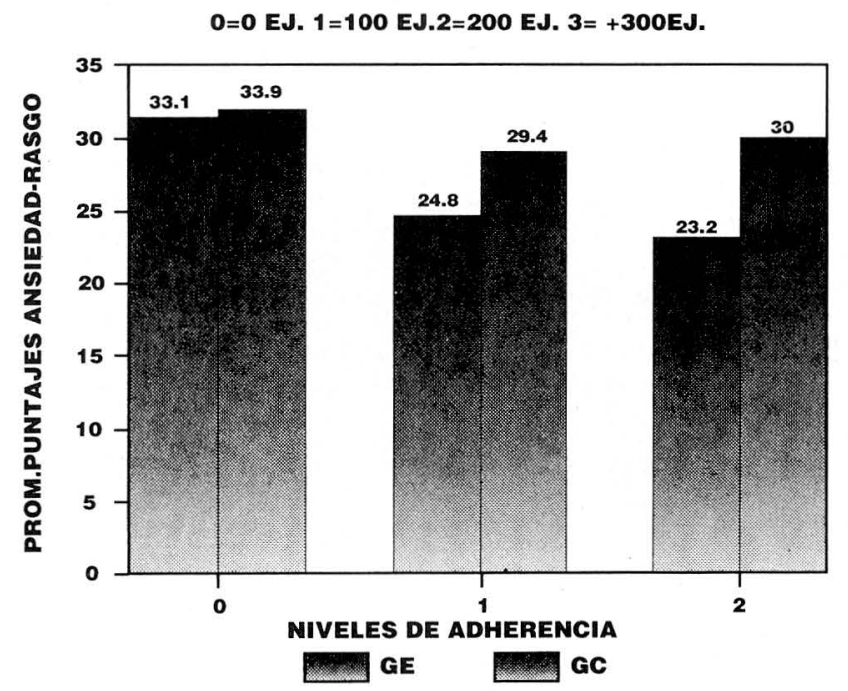

Figura 3

ADHERENCIA EN FUNCION DE LA PERSONALIDAD (NEUROTICISMO)

Niveles de adherencia en función de la personalidad para los grupos de control y experimental en la medición pre.

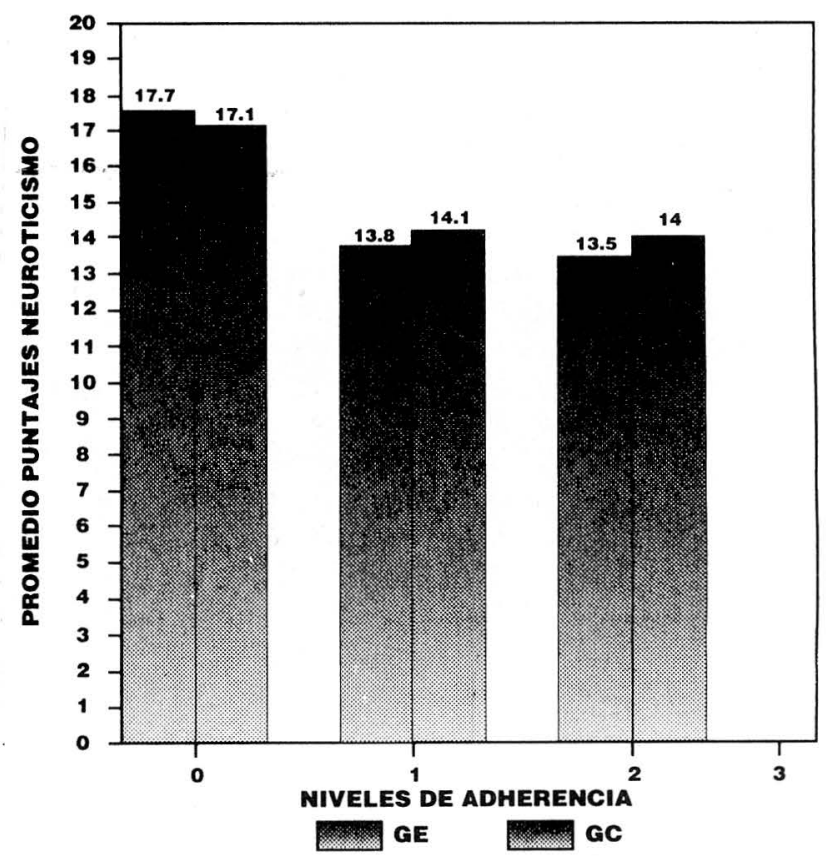

Figura 4

ASOCIACION ENTRE PERSONALIDAD Y DIAGNOSTICO

Niveles de neuroticismo de acuerdo con el diagnóstico en los grupos control y experimental.

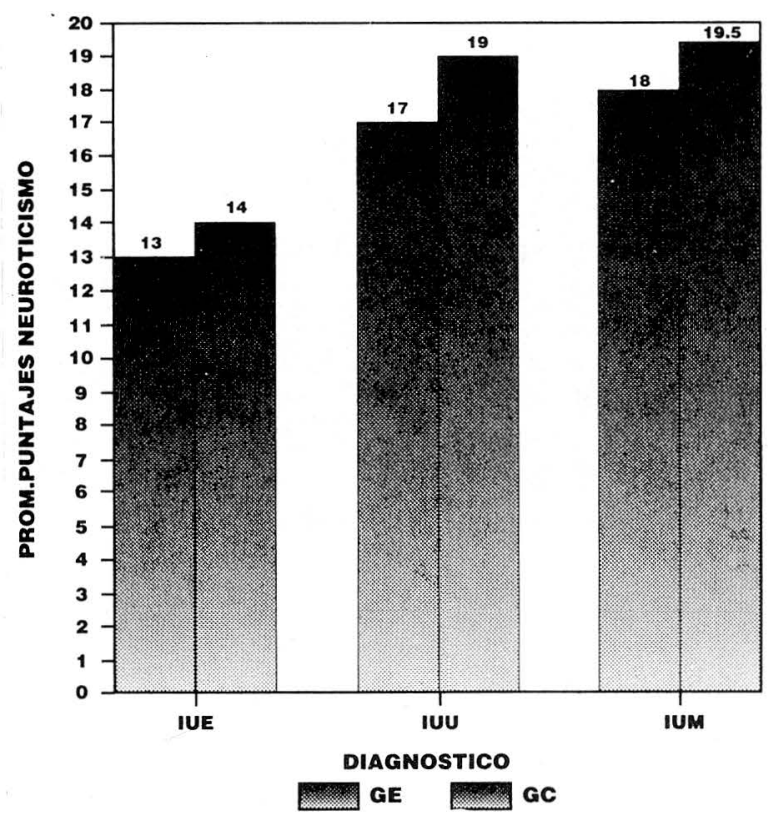


En la última semana en el GE reporta mayor concentración de sujetos en el máximo nivel de cumplimiento (300 y más ejercicios diarios), lo que ratifica la eficacia del tratamiento en la homogenización del grupo respecto del cumplimiento, eliminando las diferencias originadas en la personalidad. En el GC se sigue observando que las sujetos más cumplidoras son las menos neuróticas, pero a diferencia del GE la mayoría de las pacientes se siguen ubicando en los niveles más bajos de cumplimiento (menos de 100 ejercicios diarios) se establece entonces la eficacia y por lo tanto la necesidad de la terapia en favor de la adherencia al tratamiento.

\section{Asociación entre personalidad y el estado afectivo:}

Los resultados señalan correlaciones significativas entre niveles de neuroticismo y depresión en GE. El GC no evidencia correlaciones significativas entre las variables pero presenta la misma tendencia que el GE, a mayor depresión mayor neuroticismo correlacionan significativamente en ambos grupos.

\section{Asociación entre personalidad y estilos de afrontamiento:}

En primer lugar, se establecen asociaciones directas y significativas entre personalidad y afrontamiento:

- Fantasía y Neuroticismo $\left(0.006^{*}\right)$, evitación y Neuroticismo $(0.02 *)$.

En segundo lugar, asociaciones inversas y significativas entre: extraversión y evitación $\left(\mathrm{P}=0.03^{*}\right)$ Neuroticismo y solución de problemas $\left(\mathrm{P}=0.0002^{*}\right)$.

Por otro lado los resultados señalan asociaciones directas y significativas entre modalidades de afrontamiento: auto-culpa y fantasía $\left(\mathrm{P}=0.01^{*}\right)$. Fantasía y evitación $\left(\mathrm{P}=0.001^{*}\right)$, evitación y auto-culpa $(\mathrm{P}=0.02)$. En segunda instancia, asociaciones inversas y significativas entre las mismas variables:

Solución de problemas y fantasía $\left(\mathrm{P}=0.0001^{*}\right)$, solución de problemas y culpabilización $\left(\mathrm{P}=0.1^{*}\right)$, solución de problemas y evitación $\left(\mathrm{P}=0.001^{*}\right)$.

Los análisis para ambos grupos indican que las estrategias de afrontamiento más adaptativas son la búsqueda de soporte social y la solución de problemas, y las menos adaptativas aquellas que se centran en la autoculpabilización, la evitación y la fantasía. Estos estilos de afrontamiento desadaptativos se asocian directamente con neuroticismo.

Los resultados señalan la existencia de diferentes niveles de neuroticismo y los tres grupos diagnósticos. Las pacientes cuyo diagnóstico presenta componente de urgencia (IUU-IUM) evidencian mayores niveles de neuroticismo que aquellas que padecen incontinencia urinaria de esfuerzo (IUE) pura.

\section{Asociación entre el diagnóstico y el estado afectivo:}

Los análisis señalan la existencia de diferencias significativas entre los niveles de ansiedad-rasgo y cada uno de los diagnósticos (GE. P $=0.01 *$; GC.P=0.05+). Los más altos niveles de ansiedad se presentan en las mujeres con incontinencia de urgencia y mixta.
Tabla 1

CORRELACIONES ENTRE NEUROTICISMO Y LOS PUNTAJES DE DEPRESION Y ANSIEDAD EN LA MEDICION POST

\begin{tabular}{|c|c|c|c|}
\hline Estado Afectivo & Grupo & $\mathrm{r}$ & $\mathrm{p}$ \\
\hline Depresión & Experimental & 0.45 & $0.01^{* *}$ \\
& Control & 0.27 & N.S. \\
\hline Ansiedad & Experimental & 0.69 & $0.01^{* *}$ \\
& Control & 0.71 & $0.01^{* *}$ \\
\hline
\end{tabular}

En cuanto a la depresión, los resultados no señalan diferencias significativas con relación a cada uno de los grupos diagnósticos.

\section{Asociación entre estilos de afrontamiento y ansiedad- rasgo:}

Los resultados señalan correlaciones directas y significativas $\left(\mathrm{P}=0.008^{*}\right)$ entre los niveles de ansiedad-rasgo y las estrategias de afrontamiento concentradas en la fantasía y la evitación. Para la estrategia de afrontamiento centrada en la solución de problemas, se presentan correlaciones inversamente significativas $(\mathrm{P}=0.002 *)$.

\section{Análisis factorial de los estilos de afrontamiento:}

Los resultados indican la agrupación de los datos en tres factores:

- Uno desadaptativo I (evitación, auto-culpa y fantasía) y dos adaptativos, solución de problemas II y Apoyo Social III.

Para el factor I, la agrupación de aspectos desadaptativos en los tres niveles para su medición futura. Para el factor II, la relación inversa entre el afrontamiento centrado en la solución de ítems en cualquiera de los dos niveles para su medición. Así mismo, la relación inversa entre el apoyo social y la auto evitación, sugiere la selección de ítems en cualquiera de los dos niveles para su medición. Así mismo, la relación inversa entre el apoyo-social y la autoculpa que conforma el factor III sugiere la selección de ítems en las dos escalas.

\section{Discusión}

Dado que la adherencia al tratamiento se vio afectada tanto por el estado afectivo como por los diferentes estilos de afrontamiento y altos niveles de neuroticismo, el abordaje terapéutico debe conducir en primer lugar al reconocimiento de los diferentes patrones erróneos de pensamiento generados en este tipo de pacientes, para su posterior manejo.

Las características diferenciales encontradas de acuerdo con los tipos de diagnóstico permiten establecer propuestas de evaluación e intervención independientes, es decir, dado que pacientes con IUE pura presentan niveles más bajos de ansiedad, depresión, neuroticismo y estrategias de afrontamiento más adaptativas, dadas las correlaciones existentes entre éstas variables (a mayor neuroticismo, mayor ansiedad, mayor depresión y presencia de estrategias de afrontamiento desadaptativas), 
no se hace necesaria la aplicación de todos los inventarios para su valoración. La entrevista inicial estructurada aporta datos suficientes para la elección de las pruebas pertinentes según el caso. La intervención igualmente se adecúa de acuerdo con las características del cuadro. La tríada de auto-control, y/o la relajación autógena y/o el entrenamiento en solución de problemas, son las estrategias más apropiadas. Las pacientes responden favorablemente a estos tipos de intervenciones.

Las pacientes con Incontinencia Urinaria de Urgencia y mixta en cambio, evidencian comportamientos más desadaptativos en cuanto a depresión, ansiedad, neuroticismo y estilos de afrontamiento. Los esquemas cognitivos disfuncionales se hacen más incuestionables pudiéndose considerar como un rasgo. Esta característica obliga al uso de pruebas específicas para la identifica- ción de las diferentes dificultades que presentan. La terapia racional emotiva de Ellis, es de gran utilidad en estos casos pues el reconocimiento de patrones desadaptativos de pensamiento es la tarea más importante y así mismo la más difícil de llevar a cabo con estas pacientes.

La relajación autógena y el entrenamiento en solución de problemas pueden complementar la terapia si el caso lo amerita.

La duración de la terapia oscila entre 3 y 8 semanas de acuerdo con la gravedad de la problemática, tiempo en el cual se les proporciona el mayor apoyo posible.

Los análisis específicos para los estilos de afrontamiento arrojan conclusiones interesantes respecto a la posibilidad futura de reducir el número de ítems para la valoración e identificación de los factores que involucra.

\section{BIBLIOGRAFIA}

1. Abrams P., Blavias J., Stanton S. The standarization of terminology of Lower Urinary Tract Function. Scandinavian Journal of Urology Nephrology 1988; 144: 5.

2. Beck. A Terapia Cognitiva de la Depresión. Bilbao. 1984.

3. Bradley J., Day R. The rol the Cognition in Behavioral Medicine, Advances in Cognitive-Behavioral Research and Therapy. 1985; 4.

4. Burgio L., Robinson C., Engel B. The role of feedback in Kegel Exercises Training for Stress Urinary Incontinence. American Journal Obstetrical and Ginecology. 1986; 154(1): 58-64.

5. Cautella. Tríada de Auto Control: Un procedimiento de condicionamiento encubierto. Avances en Psicología clínica Latinoamericana. $1984 ; 3: 17-32$.

6. Davis M., McKay M y Eshelman ER. Técnicas de auto-control emocional. Barcelona: Martínez-Roca. 1985.

7. Debery y cols. Teaching Cardiac Patients to manege Medications. American Journal of Noursing. 1975; 75: 2191-2193.

8. Escobar U., Alarcón R. Relajación: aplicaciones médicas y psicológicas. Universidad industrial de Santander. Colombia. 1990.

9. Florez L. Atención Psicológica en un Servicio de Ginecología y Obstetricia. Experiencia Institucional. U.N. Facultad de Medicina. Depto. de Ginecología y Obstetricia. 1989.
10. Herzog A., Diokno A., Fulz N. Urinary Incontinence: Medical and Psichological Aspects. American Review Gerontology Geriatrics. 1948; 9: 74-79.

11. Kegel A. Progressive Resistance Exercises in the function Restauration of the perineal Muscles. American Journal of Obstetrics and Ginecology 1948; 56: 2338.

12. Lazarus, R., Folkman G., Stress apraissal and Coping. Springer. Publishing New York. 1989.

13. Lomanto A., Sánchez J. Incontinencia Urinaria de Esfuerzo: Enfoque del manejo actual. Revista Colombiana de Ginecología y Obstetricia. 1990; 39(4).

14. Mackaulay A., Stern R., Holmes H. Stanton, S. Micturituion and Mind: Psicological Factors in the Etiology and Treatment of the Simptoms in Women. British Medical Journal Clinical Research. Feb. 28249 . *6571 1987; 540-543.

15. Massur F., Andreson K. Adhesión del paciente al tratamiento médico: Un reto para la psicología de la Salud. Revista Latinoamericana de Psicología 1988; 20(10).

16. Vitallino PP., Mairu, RD., Russo J., Becker J. Raw versus Relative Scores in the assessment os coping Strategies. Journal of Behavioral Medicine 1987; 10(1): 1-18. 